\title{
How health literacy can enhance the design and conduct of clinical trials from consent to conclusion
}

\author{
Catina O’Leary ${ }^{\mathrm{a}, *}$, Chris Casey $^{\mathrm{a}}$, Diane Webb ${ }^{\mathrm{a}}$, Deborah Collyar $^{\mathrm{b}}$ and Andrew Pleasant ${ }^{\mathrm{a}}$ \\ ${ }^{a}$ Health Literacy Media, St. Louis, MO, USA \\ ${ }^{\mathrm{b}}$ Patient Advocates in Research, San Francisco, CA, USA
}

\begin{abstract}
Health literacy research and interventions have provided multiple tools to improve communication between professionals and patients in clinical contexts for many years. Despite the reality that many patients participate in clinical trials in conjunction with standard medical care, only recently have efforts extended to address and improve the health literacy of both clinical trial researchers and participants. To date, the primary focus of health literacy activities in clinical trials has centered on communicating trial results to trial participants. This report describes the opportunities and strategies necessary to layer health literacy activities across the clinical trial process from consent to conclusion.
\end{abstract}

Keywords: Health literacy, clinical trials, plain language, science communication

\section{Overview of health literacy in clinical trials}

Health literacy initiatives initially focused on patients and caregivers who did not understand medical professionals. More recently, health literacy has started to address how medical and public health professionals fail to clearly communicate with people in clinical contexts.

However, the authors believe health literacy has a larger role to play in health and well-being. One important area the authors seek to expand is to introduce health literacy within the institutions and practices of scientific research, especially clinical trials, to enhance the public's understanding and engagement in science and clinical trials.

Clinical trials face a host of significant communication challenges. These challenges are often found in the deeper meaning of words like diversity, recruitment, informed, consent, retention, engagement, participation, equity, evidence, and reporting. The words and actions each word represents can undermine a successful clinical trial. As a result, health literacy has a great and largely untapped potential to renew and reaffirm the power of clinical trials to advance health and well-being in practical, equitable, and ethical ways.

\footnotetext{
*Corresponding author: Catina O’Leary, President \& CEO, Health Literacy Media, 911 Washington Avenue, Suite 625, St. Louis MO, USA. E-mail: coleary@healthliteracy.media.
} 
Health Literacy Media (HLM) believes health literacy can make a positive and productive difference to clinical trials in terms of:

- Recruitment

- Diversity

- Informed consent

- Retention

- Engagement

- Equity

- Results, including communication of results to participants

- Evaluation

- Overall efficiency and effectiveness

More broadly, whether patients enroll in a clinical trial or are engaged in general healthcare on a broader level, diverse issues often converge to define the challenges in the relationships between medical professionals, health research, health systems, science, and society. In 1966, more than 75 percent of Americans had great confidence in medical leaders, which has declined to around 34 percent [1]. Today's health and medical systems face a crisis in confidence they are not prepared to effectively respond to and frequently are not fully aware of the breadth and depth of the calamity. The crisis is caused by the relatively narrow focus of professional training coupled with the significant financial pressures health care systems face and a resulting pressure on medical staff - all within an uncertain health economic and policy context.

In response, HLM uses health literacy as a mechanism to improve communication about and within health and medicine in order to rebuild public support for medicine, medical professionals, medical research, science, clinical trials, and health systems. In order to succeed, this effort must address a range of activities from research to sick care to prevention. HLM believes health literacy can inform and foster a necessary transformation to a healthier and more productive relationship between generally non-scientific publics and the people and institutions within health and medicine. Eventually, a shift can reverse the ongoing erosion of the public trust in the leaders of health, medical, and health care systems.

Clinical trials provide one of the most challenging contexts and case studies in which to apply the evidence-based strategy and practices of health literacy. All too often, health care professionals hear about a clinical trial being misunderstood by its participants, the press, or the public. For example, a lack of significant findings (statistically and/or clinically) in a clinical trial is often interpreted as a failure. Instead, the lack of statistical or clinically meaningful findings adds to knowledge, specifically regarding what not to pursue among specific study populations. This is just one small, yet important, example of the public misunderstanding of the processes and goals of clinical trials. HLM suggests the misunderstandings are due, in part, to how people and institutions who manage clinical trials talk about their own efforts and goals - especially during the recruitment process.

To improve public understanding, governments around the world are simultaneously working to implement new approaches, guidelines, and rules with the ultimate goal to increase the relevance and use of clinical trials.

While a well-established approach to communicate clinical trials to the public is the National Library of Medicine's website ClinicalTrials.gov, its information and data are not targeted toward lay audiences. Deborah Zarin M.D., the former director of ClinicalTrials.gov, acknowledged ClinicalTrials.gov information is provided so: "the results can be understood by an educated reader of the medical literature" [3]. 
However, the audiences many health care agencies need to reach are rarely the educated readers of medical literature. To put this another way, the diffusion of health literacy and numeracy skills (the understanding of quantitative information) is limited - especially within underserved geographic areas and populations [4-12]. Moreover, health literacy-based misunderstandings occur among well-educated people. Recent research suggests physiological shock impacts cognition regardless of a person's educational or health literacy levels when they are faced with serious medical diagnoses and diseases [13].

Despite all these potential barriers, clinical trial participants want to learn, and have the right to learn, the results of clinical trials - especially when they directly participated in that trial $[14,15]$. Providing those results in a health literate manner can increase participants' feelings of value and improve the likelihood they will participate in future trials [16,17].

Thus, there is an unprecedented urgency to address the public understanding of science, specifically clinical trials. Health communication companies with a focus on health literacy, such as HLM, are uniquely positioned to lead efforts to integrate best evidence health literacy and plain language practices into the clinical trial process. While HLM is based in the United States, we work around the world.

Advances in international policy that mandate the creation of readable and usable clinical trial summaries are driving HLM's efforts in the public communication of clinical trial information. The work reported here is based on only one program in HLM's portfolio, the Clearly Communicating Clinical Trials program, and only details initial experiences in applying health literacy principles to public communication of clinical trial information. The remaining sections of this report will briefly: (1) contextualize the international policy that served as the impetus for HLM's focus on plain language clinical trial summaries; (2) discuss the potential impact of health literacy on the clinical trial process; and (3) provide one program example and key initial learnings.

\section{European Medicines Agency mandate to create readable and usable clinical trial summaries of 2017}

The currency of the issue of health literacy in clinical trial communication partially stems from a European Medicines Agency (EMA) 536/2014 regulation, and its further clarification published in a 2017 consultation document. The EMA regulations outline the need for sponsors of clinical trials to produce socalled 'plain language' summaries of clinical trials [18-20]. The stated purpose of plain language clinical trial summaries is to provide clear information that people can use when making health decisions. More practically, the newly required summaries are discussed as a hopefully effective way to report on what occurred within a clinical trial to the participants.

In turn, the EMA's attempt to bring the reporting of clinical trial results into a more public space (based on both regulatory and ethical reasons), opens the door for trial participants and the lay public to enter a complex scientific communication sphere that historically has required medical background knowledge, familiarity with common research practices, a large clinical vocabulary, as well as the skills and access to learn, and apply the meaning of newly encountered clinical and scientific jargon.

\section{How health literacy can impact clinical trials}

As previously mentioned, health literacy can enhance every stage in the process of designing, conducting, and reporting on a clinical trial. To explain how this occurs, the authors will first introduce exactly what health literacy is - and what it is not. 
While there are many current definitions of health literacy, in practice there is some agreement that health literacy helps everyone (e.g. the public at large and health and medical professionals) better communicate complex information about health and wellbeing in a way that elevates mutual understanding and encourages informed decision-making [2]. Hypothetically, better health literacy nurtures more informed consent among participants to join clinical trials as well as fosters a dialogue about the mutual goals of participants (and other stakeholders) when a new medical drug or technology is introduced.

Health literacy is not is a panacea, however. Health literacy requires hard work, close attention to detail, a plan to move from knowledge acquisition to action, and - most importantly - direct and honest engagement with each desired audience. Except for recruitment, informed consent, and recent public clinical trial summaries, the testing and improvement of the actual communication processes and content with clinical trial participants largely has been neglected, unnoticed, and underappreciated. The vast amount of what should be continual communication between clinical trial staff and participants has largely focused on one-way communication that often fails to make sure people arrive for their appointments on time and to report any adverse events they may have experienced, let alone seeking more information about experiences, preferences, and well-being.

At the heart of health literacy is a new-found respect for the expertise that people have in their own lives. While participants may not be research scientists, physicians, nurses, public health experts, molecular scientists, geneticists, or biologists, participants know their life better than any of these experts. Embracing that reality can shift communication efforts to bring a new level of awareness and respect of the important role participants should play in clinical trial processes.

Using the EMA guidelines, some health organizations have started to adopt plain language principles - to help participants understand exactly what did (or did not) occur within a clinical trial. While this is an important first step, it is incomplete. Patient understanding does not mean trust is established and trust alone does not always result in desired patient action. On the other hand, health literacy can target and produce informed understanding, trust, and therapeutic behavioral change. The challenge is how to best implement health literacy practices in clinical trial settings as well as the venues that report trial findings.

\section{An opportunity of health literacy in clinical trials: The example of result summaries}

Health literacy strives to explain health conditions and health care concepts in language that most people can understand and use. While this seems straightforward, biopharma companies are more accustomed to communicating directly with providers rather than patients. As a result, biopharma firms are understandably wary about how to engage in patient and public communication in a respectful and non-promotional way. Yet, some of the communication gaps in past practices now need to be addressed, as biopharma companies (who are engaged in EMA-oversight clinical trials) are required to communicate clinical trial results within public summaries.

To date, some companies have recognized the need for health literate clinical trial result summaries, which is a step in the right direction. Yet, obtaining a shared understanding of clinical trial results is only a small piece of health literacy. To be truly health literate, clinical trial information also has to move beyond plain language - and contain a context that an audience can relate to, rather than simply using words that meet an arbitrary reading level guideline (such as 5th or 8th grade levels).

Consequently, a health literate approach to communicating the results of a clinical trial is a process that, ideally, begins at a drug's protocol development and continues throughout the entire clinical trial process, culminating in the delivery of a summary of results. HLM suggests a comprehensive effort should include: 
- The evidence-based best practices of plain language in the choice of language(s) to reach the desired level of reading ease and preferred language of the audience

- The evidence-based best practices of health literacy to substitute plain for scientific language to improve readers' skills, vocabulary, and knowledge. This extends the content beyond a simple reporting of data to assure that data are relevant and useful to people's lives.

- Continual input and revisions from multiple perspectives - from clinical trial staff to participants to plain language and health literacy experts. This includes:

${ }^{\circ}$ A process that produces summaries tailored to the content and context of each clinical trial.

${ }^{\circ}$ An active review process engaging members of the intended audiences, especially people with the condition or disease focused on in the result summary.

${ }^{\circ}$ The latter should occur before publication in order to assure effectiveness and usability.

- An evaluation of the effects of the summary upon the desired audiences and upon all the other audiences involved in a clinical trial process and the reporting of its results.

While the authors are using result summaries as the primary example, health literate attributes are critically important within other aspects of the clinical trial process from protocol development to recruitment, informed consent, and the reporting of results. When this entire process is conducted rigorously from day one of a clinical trial, result summaries will often - but not always - include:

- Visual explanations of complex concepts, using charts, tables, and drawings

- Analogies people can relate to based on their everyday lives

- Clear language that explains complex science in accurate and understandable ways to help teach people the words they need to use to successfully communicate with medical staff

- Publications tailored to the diverse channels that people use in their lives (e.g. social media, internet, print, and video)

- A clinical trial staff that is prepared to communicate the trial's results directly to participants.

Successful, comprehensive public and patient communication also recognizes the value of tailoring content to audiences, which contrasts with the use of templates to communicate to the public. Tailoring is operationally defined as the process of personalizing messages for individuals and audiences based on their beliefs, traits, or abilities [21]. Tailoring can help weave context throughout materials. While a template approach to communication assumes all clinical trial results can fit within the same template, the effectiveness of tailoring lies in its ability to create potentially greater relevance to each intended audience [22].

Yet, the EMA guidelines have been widely used to create templates for the diverse sections a trial result summary should contain. Unfortunately, some communicators have taken those guidelines too literally and assume the order in which information is presented must follow the order they are listed in within the guidelines. In addition, strict adherence to template styles can result in a confusing focus on outcomes. For example, early phase 2 studies largely report data on safety without outcomes, while later phase studies largely report outcomes and also safety. Thus, a template that prioritizes outcomes would not be appropriate for a study largely focused on safety and vice versa. Conversely, a tailored approach - if taken too far - could mean each result summary would begin with a blank page, require extensive research of the target audience in advance, and quickly become a labor-intensive process that would outstrip the resources dedicated to creating the summary.

To remedy, HLM suggests a balanced approach, whereby a template may be used to create the initial draft of a clinical trial summary, which can then be tailored through a user testing process. Thus, HLM 
developed a clinical trial summary template built on the principles of: patient-centered communication; plain language; health literacy; logical idea structure; and an explanation of background knowledge to clearly explain the need and desired outcomes of a clinical trial. HLM then used a focus group to test the clinical trial summary template to create a more tailored clinical trial communication. Focus groups are considered to be a standard to assess an audience's preferences and their understanding of documents.

In this early development stage of HLM's clinical trial summary development process, two audience groups were identified and focus groups were conducted to develop and test a clinical trial result summary template $[23,24]$. One group was recruited from an adult literacy education program and included adults with low literacy. The second group of participants was recruited from people who had the condition the template draft focused upon (breast cancer). In addition to testing the template, overall, the focus groups helped HLM identify and better understand the impact of language that might be unfamiliar to people who are more and less experienced with a particular medical condition. In addition, the focus groups suggested lay audiences appreciated more background information about a disease, as they may have less knowledge about medical conditions and treatments. This contrasted with breast cancer patients, who sometimes preferred more detail about treatments, side effects, and results than members of the general public [25]. Both lay audiences and people who are more directly impacted by a disease/illness sought additional context about what the results mean for patients as they consider treatment or other medical decisions. Thus, the value of tailoring became as apparent as the value of a template approach in an early, formative research process.

\section{Key guidelines to creating a clinical trial summary based on HLM's research and experience}

Fundamentally, all successful communication of complex information shares similar characteristics regardless of the specific content or audience - therein lies HLM's development and use of templates. Yet, all audiences for the communication of complex information are not the same - therein lies HLM's development and use of a research-based approach to engage audiences directly and tailor information to their needs within a template.

HLM suggests there are several principles to produce a usable and effective clinical trial summary. More specifically, HLM suggests:

- Above all, stay true to the science

${ }^{\circ}$ In very practical terms, this means to never include any information that is not contained within the core reports produced by the clinical trial team.

- Quickly relay the main point of the study and any findings

${ }^{\circ}$ State the main need for and findings of the study up front. This produces a flow of information that is generally the reverse order of a traditional journal and regulatory reporting in which the outcomes are often found near the end of an article or report.

- Frame the findings in a useful way for the main audiences

- This can mean presenting information based as an answer to the main questions people have. Those questions are often used as headers for each 'chunk' of information within a summary.

${ }^{\circ}$ Describe who participated in a clinical trial in as clear and complete a style as the original scientific documents. 
- Highlight the necessary cautions to using scientific information within an informed decision-making process

${ }^{\circ}$ Be clear that a health care decision should not be based on the findings of a single clinical trial and consulting health care professionals is always a vital part of an informed decision-making process.

In practice, HLM's approach and suggested principles are based upon effective methods to create clinical trial summaries. HLM acknowledges their suggested approach is not simple, and requires skilled personnel, health literacy training, and a meaningful investment of their time and energy.

For instance, HLM suggests a clinical trial summary creation process begin with a thorough review of the documentation available about the clinical trial as well as becoming familiar with the condition that each clinical trial focuses upon. Built into this process should be an opportunity to directly discuss with the scientific team the understandings and questions about the clinical trial. The latter direct communication, while invaluable, also can identify different approaches and perceptions of the value of communicating scientific information, and how information should be communicated between the writing of a summary and the researchers who designed and conducted the entire clinical trial.

HLM notes an enduring paradigm within science fosters a communication approach that sometimes is at odds with communicating directly with non-scientific audiences. For example, the use of scientific jargon has several underlying components, which frequently include:

- The language, especially the vocabulary, unique to a particular trade, profession, or group

- Unclear or meaningless (to certain audiences) talk or writing

- A discussion section or writing that people do not understand

- Language that is characterized by uncommon or pretentious vocabulary and convoluted syntax that is often vague in meaning.

While jargon enables scientists to more efficiently communicate with each other as they develop a shared understanding of jargon-derived meanings, the public rarely shares similar insights. The resulting gaps produce a choice about communication priorities that HLM suggests should be resolved on the basis of developing public understanding. Ultimately, the culture and needs of the audience must be prioritized to produce an understandable and usable clinical trial summary. The latter approach additionally favorably shifts the culture of science toward public engagement and away from a perspective that separates science from society.

\section{Wrapping up}

So, why does this all matter? Especially, why should this matter to an industry whose main goal is to create successful and profitable new approaches to medicine?

Clinical trials should not occur without honest and sincere public engagement. If done well, clinical trial summaries offer opportunities to engage the public and patients in new ways and helps create a more efficient clinical trial process. Overall, clinical trial summaries can help the pharmaceutical industry, researchers, clinical practitioners, hospitals and clinics, as well as government and non-governmental health agencies:

- Show respect to trial participants

- Help health care providers keep up with research advances

- Show how clinical trial participation can lead to better medical solutions 
- Help people quickly learn more about new medical conditions, and what to do about it

- Help people develop the critical questions they need to make decisions about their health.

Public clinical trial summaries also present an opportunity to create information from a perspective that can engage and involve people in their own medical care, while creating goodwill and a better understanding of the companies, agencies, and scientists who conduct medical research.

The authors believe that health literacy offers significant potential to improve and advance clinical trials when health literacy strategies are used throughout the entire clinical trial process. The latter can occur at every phase of a clinical trial to produce the best possible outcomes for companies, providers, and patients.

For all audiences, the application of health literacy to clinical trial processes can promote a shared understanding of the intent of a clinical trial, the outcomes of the clinical trial, as well as build trust in the clinical trial process. The application of health literacy processes also potentially accelerates the public adoption of clinical trial findings, which could improve patient adherence.

Finally, each step within this process needs to explain research ethics to produce more public trust in the outcomes of clinical trials. Based on our engagement in and understanding of the clinical trial process, the next steps involve extending the application of health literacy best practices into all aspects of the clinical trial process - from the development phase, to recruitment and retention efforts, and across dissemination and public communication. The authors' experiences, to date, suggest that undergirding all aspects of the clinical trial process with health literacy approaches will foster more sustainable science, the sustainability of organizations sponsoring and conducting clinical trials, and the sustainability of the health and wellbeing of patients and the public.

\section{References}

[1] R. Blendon, J. Benson and J. Hero, Public trust in physicians - U.S. medicine in international perspective, New Engl J Med 371: (2014), 1570-1572. doi:10.1056/NEJMp1407373.

[2] A. Pleasant, R.E. Rudd, C. O’Leary, M.K. Paasche-Orlow, M.P. Allen, W. Alvarado-Little et al. Considerations for a New Definition of Health Literacy. National Academy of Medicine, Washington DC, 2016. doi:10.31478/201604a.

[3] D.A. Zarin, T. Tse, R.J. Williams, R.M. Califf and N.C. Ide, The ClinicalTrials.gov results database-update and key issues, New Engl J Med 364(9) (2011), 852-860. doi:10.1056/NEJMsa1012065.

[4] K.L. Jacobson and R.M. Parker, Health Literacy Principles: Guidance for Making Information Understandable, Useful, and Navigable National Academy of Sciences, 2014, http://www.iom.edu/Global/Perspectives/2014/ HealthLiteracyGuidance.aspx.

[5] The Health Literacy Style Manual MAXIMUS Center for Health Literacy, 2005, http://www.coveringkidsandfamilies.org/ resources/docs/stylemanual.pdf.

[6] J.R. Ridpath, S.M. Greene and C.J. Wiese, PRISM Readability Toolkit, 3rd ed. Group Health Research Institute, Seattle, 2007.

[7] B.J. Zikmund-Fisher, The right tool is what they need, not what we have: A taxonomy of appropriate levels of precision in patient risk communication, Medical Care Research and Review 70(1 suppl) (2013), 37S-49S. doi: $10.1177 / 1077558712458541$.

[8] G.H. McLaughlin, SMOG grading: A new readability formula, Journal of Reading 12(8) (1969), 639-646.

[9] M. Kutner, E. Greenberg, Y. Jin and C. Paulsen, The Health Literacy of America's Adults: Results from the 2003 National Assessment of Adult Literacy. U.S. Department of Education. National Center for Education Statistics, Washington, DC, 2006, p. 483

[10] D.E. Nelson, B.W. Hesse and R.T. Croyle, Making Data Talk: A Workbook National Institute of Health Publication, 2011, 11-7724.

[11] Federal Plain Language Guidelines. Revision 1, 2011. http://www.plainlanguage.gov/howto/guidelines/FederalPL Guidelines/FederalPLGuidelines.pdf. 
[12] Institute of Medicine. Health Literacy and Numeracy: Workshop Summary. The National Academies Press, Washington, DC, 2014. doi:10.17226/18660.

[13] R.P. Kessels, Patients' memory for medical information, JRSM Open 96(5) (2003), 219-222. doi:10.1258/jrsm.96.5.219.

[14] S.Y. Moorcraft, C. Marriott, C. Peckitt, D. Cunningham, I. Chau, N. Starling, D. Watkins and S. Rao, Patients' willingness to participate in clinical trials and their views on aspects of cancer research: Results of a prospective patient survey, Trials 17 (2016), 17. doi:10.1186/s13063-015-1105-3.

[15] D.I. Shalowitz and F.G. Miller, Communicating the results of clinical research to participants: attitudes, practices, and future directions, PLoS Med 5(5) (2008), e91. doi:10.1371/journal.pmed.0050091.

[16] A.H. Partridge, A.C. Wolff, P.K. Marcom et al., The impact of sharing results of a randomized breast cancer clinical trial with study participants, Breast Cancer Res Treat 115(1) (2009), 123-129. doi:10.1007/s10549-008-0057-7.

[17] A. Sood, K. Prasad, L. Chhatwani, E. Shinozaki, S.S. Cha, L.L. Loehrer and D.L. Wahner-Roedler, Patients' attitudes and preferences about participation and recruitment strategies in clinical trials, Mayo Clin Proc 84(3) (2009), $243-247$. doi:10.1016/S0025-6196(11)61141-5.

[18] http://www.ema.europa.eu/ema/index.jsp?curl=pages/regulation/general/general_content_000629.jsp. Retrieved October $13,2017$.

[19] The European Parliament and the Council of the European Union. Regulation (EU) No 536/2014 of the European Parliament and of the Council of 16 April 2014 on clinical trials on medicinal products for human use, and repealing Directive 2001/20/EC, Official Journal of the European Union (2014) L158/1-76. https://ec.europa.eu/health//sites/health/files/files/eudralex/vol-1/reg_2014_536/reg_2014_536_en.pdf.

[20] European Commission. Summaries of clinical trial results for laypersons: Recommendations of the expert group on clinical trials for the implementation of regulation (EU) No 536/2014 on clinical trials on medicinal products for human use, European Medicines Agency website. https://ec.europa.eu/health/sites/health/files/files/eudralex/ vol-10/2017_01_26_summaries_of_ct_results_for_laypersons.pdf. Retrieved 10/13/2017.

[21] M.W. Kreuter, V.J. Strecher and B. Glassman, One size does not fit all: The case for tailoring print materials, Ann Behav Med 21: (1999), 276-283. doi:10.1007/BFo2895958.

[22] J.D. Jenson, A.J. King, N. Carcioppolo and L. Davis, Why are tailored messages more effective? A multiple mediation analysis of a breast cancer screening intervention, J Commun 62(5) (2012), 851-868. doi:10.1111/j.1460-2466.2012.01668.x.

[23] I. McLafferty, Focus group interviews as a data collecting strategy, $J$ Adv Nurs 48(2) (2004), 187-194. doi:10.1111/j.13652648.2004.03186.x.

[24] E.J. Halcomb, L. Gholizadeh, M. DiGiacomo, J. Phillips and P.M. Davidson, Literature review: Considerations in undertaking focus group research with culturally and linguistically diverse groups, J Clin Nurs 16(6) (2007), 1000-1011. doi:10.1111/j.1365-2702.2006.01760.x.

[25] A.H. Partridge and E.P. Winer, Sharing study results with trial participants: Time for action, J Clin Oncol 27(6) (2009), 838-839. doi:10.1200/JCO.2008.20.0865. 